\title{
On the Existence of Global Weak Solutions for a Weakly Dissipative Hyperelastic Rod Wave Equation
}

\author{
Haibo Yan, Ls Yong, Yu Yang, and Yang Wang \\ Department of Mathematics, Southwestern University of Finance and Economics, Chengdu 611130, China \\ Correspondence should be addressed to Haibo Yan; wengxiang00@163.com
}

Received 6 July 2014; Accepted 16 August 2014; Published 2 September 2014

Academic Editor: De-Xing Kong

Copyright (C) 2014 Haibo Yan et al. This is an open access article distributed under the Creative Commons Attribution License, which permits unrestricted use, distribution, and reproduction in any medium, provided the original work is properly cited.

Assuming that the initial value $v_{0}(x)$ belongs to the space $H^{1}(R)$, we prove the existence of global weak solutions for a weakly dissipative hyperelastic rod wave equation in the space $C([0, \infty) \times R) \bigcap L^{\infty}\left([0, \infty) ; H^{1}(R)\right)$. The limit of the viscous approximation for the equation is used to establish the existence.

\section{Introduction}

This paper focuses on the study of the weakly dissipative model

$$
\begin{aligned}
& v_{t}-v_{t x x}+\partial_{x} f(v)+\lambda\left(v-v_{x x}\right) \\
& =2 \alpha v_{x} v_{x x}+\alpha v v_{x x x}+\beta v^{2 m} v_{x x},
\end{aligned}
$$

where $\alpha>0, \beta \geq 0, \lambda \geq 0, f(v)$ is a polynomial with order $n$, and $m$ is a nonnegative integer. If $f(v)=2 k v+$ $(3 / 2) v^{2}, \alpha=1, \beta=0$, and $\lambda=0$, (1) becomes the CamassaHolm equation [1]. When $f(v)=(3 / 2) v^{2}$ and $\lambda=\beta=0$, (1) is turned into the hyperelastic rod wave equation [2]. Since the terms $\lambda\left(v-v_{x x}\right)$ and $\beta v^{2 m} v_{x x}$ appear in the equation, here we call (1) a weakly dissipative hyperelastic rod wave equation.

In order to link with previous researches in the field, we state here several works on the existence of global weak solution for the Camassa-Holm equation $(\mathrm{CH})$ and a generalized hyperelastic rod wave equation (or a generalized Camassa-Holm equation). Under the sign condition of the initial value, Constantin and Escher [3] obtained the existence and uniqueness results for the global weak solutions of Camassa-Holm equation (also see Constantin and Molinet [4]). Xin and Zhang [5] proved the existence of the global weak solution for the Camassa-Holm equation in the energy space $H^{1}(R)$ without adding the sign conditions on the initial value. Coclite et al. [6] used the analysis in [5] and established the existence of global weak solutions for a generalized hyperelastic rod wave equation (or a generalized Camassa-Holm equation); namely, $\lambda=\beta=0$ in (1). For the global or local solutions of the Camassa-Holm equation and other partial differential equations, the reader is referred to [7-12] and the references therein.

The objective of this paper is to extend parts of works made by Coclite et al. [6]. We investigate the existence of global weak solutions for the weakly dissipative hyperelastic rod wave equation (1) in the space $C([0, \infty) \times$ $R) \bigcap L^{\infty}\left([0, \infty) ; H^{1}(R)\right)$. Using the limit of viscous approximations for the equation and several estimates derived from the equation itself, we establish the existence of the global weak solution by merely assuming the initial value in the space $H^{1}(R)$.

This paper is organized as follows. The main result is stated in Section 2. In Section 3, we present the viscous problem and establish several estimates of the problem. Namely, we give proofs of an upper bound, a higher integrability estimate, and some basic compactness properties for the viscous approximations. Strong compactness about the derivative of solutions for the viscous approximations is proved in Section 4, where the proof of the main result is finished. 


\section{Main Result}

We write the Cauchy problem for (1):

$$
\begin{gathered}
v_{t}-v_{t x x}+\partial_{x} f(v)+\lambda\left(v-v_{x x}\right) \\
=2 \alpha v_{x} v_{x x}+\alpha v v_{x x x}+\beta v^{2 m} v_{x x} \\
v(0, x)=v_{0}(x)
\end{gathered}
$$

which is equal to the form

$$
\begin{gathered}
v_{t}+\alpha v v_{x}+\lambda v+\frac{\partial Q}{\partial x}=0, \\
\frac{\partial Q}{\partial x}=\Lambda^{-2} \partial_{x}\left[f(v)-\frac{\alpha}{2} v^{2}+\frac{\alpha}{2} v_{x}^{2}-\beta v^{2 m} v_{x}\right] \\
+2 m \beta \Lambda^{-2}\left[v^{2 m-1} v_{x}^{2}\right], \\
v(0, x)=v_{0}(x),
\end{gathered}
$$

where the operator $\Lambda^{2}=1-\left(\partial^{2} / \partial x^{2}\right)$ and

$$
\Lambda^{-2} h(x)=\frac{1}{2} \int_{R} e^{-|x-y|} h(y) d y \quad \text { for } h(x) \in L^{2}(R) .
$$

Using problem (2), we derive the conservation law

$$
\begin{aligned}
\int_{R}\left(v^{2}+v_{x}^{2}\right) d x+2 \int_{0}^{t} \int_{R}[ & \beta(2 m+1) v^{2 m} v_{x}^{2} \\
& \left.+\lambda\left(v^{2}+v_{x}^{2}\right)\right] d x d t \\
=\int_{R}\left(v_{0}^{2}+v_{0 x}^{2}\right) d x . &
\end{aligned}
$$

Here we adopt the definition of global weak solution presented in [5].

Definition 1. A continuous function $v:[0, \infty) \times R \rightarrow R$ is said to be a global weak solution for the Cauchy problem (2) or (3) if

(a) $v \in C([0, \infty) \times R) \bigcap L^{\infty}\left([0, \infty) ; H^{1}(R)\right)$;

(b) $\|v(t, \cdot)\|_{H^{1}(R)} \leq\left\|v_{0}\right\|_{H^{1}(R)}$;

(c) $v=v(t, x)$ satisfies (3) in the sense of distributions and takes on the initial value pointwise.

Now we state the main result of this paper.

Theorem 2. If $v_{0}(x) \in H^{1}(R)$, then the Cauchy problem (2) or (3) has a global weak solution $v(t, x)$ in the sense of Definition 1. In addition, the weak solution has the following properties.

(1) There exists a positive constant $c_{0}$ depending on $\left\|v_{0}\right\|_{H^{1}(R)}$ and the coefficients of (1) such that

$$
\frac{\partial v(t, x)}{\partial x} \leq \frac{4}{\alpha t}+c_{0}, \quad \text { for }(t, x) \in[0, \infty) \times R .
$$

(2) Assume that $0<\rho<1, T>0$, and $a, b \in R$, $a<$ $b$. Then there exists a positive constant $c_{1}$ depending only on $\left\|v_{0}\right\|_{H^{1}(R)}, \rho, T, a, b$, and the coefficients of (1) such that

$$
\int_{0}^{t} \int_{a}^{b}\left|\frac{\partial v(t, x)}{\partial x}\right|^{2+\rho} d x \leq c_{1} .
$$

\section{Viscous Approximations}

Consider

$$
\phi(x)= \begin{cases}e^{1 /\left(x^{2}-1\right)}, & |x|<1 \\ 0, & |x| \geq 1\end{cases}
$$

We choose the mollifier $\phi_{\varepsilon}(x)=\varepsilon^{-1 / 4} \phi\left(\varepsilon^{-1 / 4} x\right)$ with $0<\varepsilon<$ $1 / 4$ and $v_{\varepsilon, 0}=\phi_{\varepsilon} \star v_{0}$. We know that $v_{\varepsilon, 0} \in C^{\infty}$ for any $v_{0} \in H^{s}$, $s>0$ (see [7]). In fact, we have

$$
\begin{gathered}
\left\|v_{\varepsilon, 0}\right\|_{H^{1}(R)} \leq c\left\|v_{0}\right\|_{H^{1}(R)}, \\
v_{\varepsilon, 0} \longrightarrow v_{0} \text { in } H^{1}(R),
\end{gathered}
$$

where $c$ is a positive constant.

To prove the existence of global weak solutions to the Cauchy problem (3), we will establish compactness of a sequence of smooth functions $\left\{v_{\varepsilon}\right\}_{\mathcal{E}>0}$ satisfying the viscous problem

$$
\begin{gathered}
\frac{\partial v_{\varepsilon}}{\partial t}+\alpha v_{\varepsilon} \frac{\partial v_{\varepsilon}}{\partial x}+\lambda v_{\varepsilon}+\frac{\partial Q_{\varepsilon}}{\partial x}=\varepsilon \frac{\partial^{2} v_{\varepsilon}}{\partial x^{2}} \\
\frac{\partial Q_{\varepsilon}}{\partial x}=\Lambda^{-2} \partial_{x}\left[f\left(v_{\varepsilon}\right)-\frac{\alpha}{2} v_{\varepsilon}^{2}+\frac{\alpha}{2}\left(\frac{\partial v_{\varepsilon}}{\partial x}\right)^{2}-\beta v_{\varepsilon}^{2 m} \frac{\partial v_{\varepsilon}}{\partial x}\right] \\
+2 m \beta \Lambda^{-2}\left[v_{\varepsilon}^{2 m-1}\left(\frac{\partial v_{\varepsilon}}{\partial x}\right)^{2}\right], \\
v_{\varepsilon}(0, x)=v_{\varepsilon, 0}(x) .
\end{gathered}
$$

For simplicity, throughout this paper, we let $c$ denote any positive constant which is independent of parameter $\varepsilon$.

Now we give the well-posedness result to problem (10).

Lemma 3. If $v_{0} \in H^{1}(R)$, for any $\sigma \geq 1$, there is a unique solution $v_{\varepsilon} \in C([0, \infty) \times R)$ to the Cauchy problem (10). In addition, it holds that

$$
\begin{aligned}
& \int_{R}\left(v_{\varepsilon}^{2}+\left(\frac{\partial v_{\varepsilon}}{\partial x}\right)^{2}\right) d x \\
& +2 \int_{0}^{t} \int_{R}\left[\beta(2 m+1) v_{\varepsilon}^{2 m}\left(\frac{\partial v_{\varepsilon}}{\partial x}\right)^{2}\right. \\
& \left.+\lambda\left(v_{\varepsilon}^{2}+\left(\frac{\partial v_{\varepsilon}}{\partial x}\right)^{2}\right)\right] d x d t \\
& +2 \varepsilon \int_{0}^{t} \int_{R}\left(\left(\frac{\partial v_{\varepsilon}}{\partial x}\right)^{2}+\left(\frac{\partial^{2} v_{\varepsilon}}{\partial x^{2}}\right)^{2}\right)(s, x) d x d s \\
& =\left\|v_{\varepsilon, 0}\right\|_{H^{1}(R)}^{2}
\end{aligned}
$$


or

$$
\begin{aligned}
& \left\|v_{\varepsilon}(t, \cdot)\right\|_{H^{1}(R)}^{2}+2 \varepsilon \int_{0}^{t}\left\|\frac{\partial v_{\varepsilon}}{\partial x}(s, \cdot)\right\|_{H^{1}(R)}^{2} d s \\
& \quad+2 \lambda \int_{0}^{t}\left\|v_{\varepsilon}(s, \cdot)\right\|_{H^{1}(R)}^{2} d s \\
& \quad+2 \beta(2 m+1) \int_{0}^{t} \int_{R} v_{\varepsilon}^{2 m}\left(\frac{\partial v_{\varepsilon}}{\partial x}\right)^{2} d x=\left\|v_{\varepsilon, 0}\right\|_{H^{1}(R)^{2}}^{2}
\end{aligned}
$$

Proof. If $\sigma \geq 1$ and $v_{0} \in H^{1}(R)$, we know $v_{\varepsilon, 0} \in$ $C\left([0, \infty) ; H^{\infty}(R)\right)$. Using Theorem 2.1 in [6] or Theorem 2.3 in $[10]$, we derive that problem (10) has a unique solution $v_{\varepsilon} \in C\left([0, \infty) ; H^{\infty}(R)\right)$.

Applying the first equation in problem (10), we derive

$$
\begin{aligned}
& \frac{\partial v_{\varepsilon}}{\partial t}-\frac{\partial^{3} v_{\varepsilon}}{\partial t x^{2}}+\frac{\partial f\left(v_{\varepsilon}\right)}{\partial x}+\lambda\left(v_{\varepsilon}-\frac{\partial^{2} v_{\varepsilon}}{\partial x^{2}}\right) \\
& =2 \alpha \frac{\partial v_{\varepsilon}}{\partial x} \frac{\partial^{2} v_{\varepsilon}}{\partial x^{2}}+\alpha v_{\varepsilon} \frac{\partial^{3} v_{\varepsilon}}{\partial x^{3}}+\beta v_{\varepsilon}^{2 m} \frac{\partial^{2} v_{\varepsilon}}{\partial x^{2}}+\varepsilon\left(\frac{\partial^{2} v_{\varepsilon}}{\partial x^{2}}-\frac{\partial^{4} v_{\varepsilon}}{\partial x^{4}}\right),
\end{aligned}
$$

from which we obtain

$$
\begin{aligned}
& \frac{1}{2} \frac{d}{d t} \int_{R}\left(v_{\varepsilon}^{2}+\left(\frac{\partial v_{\varepsilon}}{\partial x}\right)^{2}\right) d x+\lambda \int_{R}\left[v_{\varepsilon}^{2}+\frac{\partial^{2} v_{\varepsilon}}{\partial x^{2}}\right] d x \\
& \quad+\beta(2 m+1) \int_{R} v_{\varepsilon}^{2 m}\left(\frac{\partial v_{\varepsilon}}{\partial x}\right)^{2} d x \\
& \quad+\varepsilon \int_{R}\left(\left(\frac{\partial v_{\varepsilon}}{\partial x}\right)^{2}+\left(\frac{\partial^{2} v_{\varepsilon}}{\partial x^{2}}\right)^{2}\right) d x=0 .
\end{aligned}
$$

The proof is completed.

Using Lemma 3, we get

$$
\left\|v_{\varepsilon}\right\|_{L^{\infty}(R)} \leq\left\|v_{\varepsilon}\right\|_{H^{1}(R)} \leq\left\|v_{\varepsilon, 0}\right\|_{H^{1}(R)} \leq c\left\|v_{0}\right\|_{H^{1}(R)} .
$$

Differentiating the first equation of problem (10) with respect to $x$ and writing $\partial v_{\varepsilon} / \partial x=q_{\varepsilon}$, we obtain

$$
\begin{gathered}
\frac{\partial q_{\varepsilon}}{\partial t}+\alpha v_{\varepsilon} \frac{\partial q_{\varepsilon}}{\partial x}-\varepsilon \frac{\partial^{2} q_{\varepsilon}}{\partial x^{2}}+\frac{\alpha}{2} q_{\varepsilon}^{2}+\beta\left(v_{\varepsilon}\right)^{2 m} q_{\varepsilon}+\lambda q_{\varepsilon} \\
=f\left(v_{\varepsilon}\right)-\frac{\alpha}{2} v_{\varepsilon}^{2} \\
-\Lambda^{-2}\left[f\left(v_{\varepsilon}\right)-\frac{\alpha}{2}\left(v_{\varepsilon}^{2}-q^{2}\right)-\beta\left(v_{\varepsilon}\right)^{2 m} q_{\varepsilon}\right. \\
\left.+2 m \beta \partial_{x}\left[\left(v_{\varepsilon}\right)^{2 m-1} q_{\varepsilon}^{2}\right]\right]=P_{\varepsilon}(t, x) .
\end{gathered}
$$

Lemma 4. Let $0<\rho<1, T>0$, and $a, b \in R$, $a<b$. Then there exists a positive constant $c_{1}$ depending only on $\left\|v_{0}\right\|_{H^{1}(R)}$, $\rho, T, a, b$, and the coefficients of (1), but independent of $\varepsilon$, such that the space higher integrability estimate holds:

$$
\int_{0}^{T} \int_{a}^{b}\left|\frac{\partial v_{\varepsilon}(t, x)}{\partial x}\right|^{2+\rho} d x \leq c_{1},
$$

where $v_{\varepsilon}=v_{\varepsilon}(t, x)$ is the unique solution of problem $(10)$.
Proof. The proof is a variant of the proof presented in Xin and Zhang [5] (or see Coclite et al. [6]). Let $\chi \in C^{\infty}(R)$ be a cut-off function such that $0 \leq \chi \leq 1$ and

$$
\chi(x)= \begin{cases}1, & \text { if } x \in[a, b], \\ 0, & \text { if } x \in(-\infty, a-1] \cup[b+1, \infty) .\end{cases}
$$

Considering the map $\omega(\xi):=\xi(1+|\xi|)^{\rho}, \xi \in R, 0<\rho<1$, and observing that

$$
\begin{aligned}
\omega^{\prime}(\xi)= & (1+(1+\rho)|\xi|)(1+|\xi|)^{\rho-1} \\
\omega^{\prime \prime}(\xi)= & \rho \operatorname{sign}(\xi)(1+|\xi|)^{\rho-2}(2+(1+\rho)|\xi|), \\
= & \rho(1+\rho) \operatorname{sign}(\xi)(1+|\xi|)^{\rho-1} \\
& +(1-\rho) \rho \operatorname{sign}(\xi)(1+|\xi|)^{\rho-2}
\end{aligned}
$$

we have

$$
\begin{aligned}
& |\omega(\xi)| \leq|\xi|+|\xi|^{1+\rho}, \\
& \left|\omega^{\prime}(\xi)\right| \leq 1+(1+\rho)|\xi|, \quad\left|\omega^{\prime \prime}(\xi)\right| \leq 2 \rho \\
& \xi \omega(\xi)-\frac{1}{2} \xi^{2} \omega^{\prime}(\xi) \\
& \quad=\frac{1-\rho}{2} \xi^{2}(1+|\xi|)^{\rho}+\frac{\rho}{2} \xi^{2}(1+|\xi|)^{\rho-1} \\
& \quad \geq \frac{1-\rho}{2} \xi^{2}(1+|\xi|)^{\rho} .
\end{aligned}
$$

Differentiating the first equation of problem (10) with respect to $x$ and setting $v=v_{\varepsilon}$ and $\partial v_{\varepsilon} / \partial x=q_{\varepsilon}=q$ for simplicity, we obtain

$$
\begin{aligned}
& \frac{\partial q}{\partial t}+\alpha v \frac{\partial q}{\partial x}-\varepsilon \frac{\partial^{2} q}{\partial x^{2}}+\frac{\alpha}{2} q^{2}+\beta\left(v_{\varepsilon}\right)^{2 m} q+\lambda q \\
&=f(v)-\frac{\alpha}{2} v^{2}-\Lambda^{-2}\left[f(v)-\frac{\alpha}{2}\left(v^{2}-q^{2}\right)-\beta v^{2 m} q\right. \\
&\left.+2 m \beta \partial_{x}\left[v^{2 m-1} v_{x}^{2}\right]\right]=P_{\varepsilon}(t, x) .
\end{aligned}
$$

Multiplying (22) by $\chi \omega^{\prime}(q)$ and integrating over $\Pi_{T}:=[0$, $T] \times R$, we get

$$
\begin{aligned}
& \int_{\Pi_{T}} \alpha \chi(x) q \omega(q) d t d x-\frac{\alpha}{2} \int_{\Pi_{T}} q^{2} \chi(x) \omega^{\prime}(q) d t d x \\
& =\int_{R} \chi(x)(\omega(q(t, x))-\omega(q(0, x))) d x \\
& \quad-\int_{\Pi_{T}} \alpha v \chi^{\prime}(x) \omega(q) d t d x \\
& \quad+\varepsilon \int_{\Pi_{T}} \frac{\partial q}{\partial x} \chi^{\prime}(x) \omega^{\prime}(q) d t d x \\
& \quad+\varepsilon \int_{\Pi_{T}}\left(\frac{\partial q}{\partial x}\right)^{2} \chi(x) \omega^{\prime \prime}(q) d t d x
\end{aligned}
$$




$$
\begin{aligned}
& +\int_{\Pi_{T}}\left[\beta v^{2 m}+\lambda\right] q \chi(x) \omega^{\prime}(q) d t d x \\
& -\int_{\Pi_{T}} Q_{\varepsilon}(t, x) \chi(x) \omega^{\prime}(q) d t d x .
\end{aligned}
$$

Using (21) yields

$$
\begin{gathered}
\int_{\Pi_{T}} \alpha \chi(x) q \omega(q) d t d x-\frac{\alpha}{2} \int_{\Pi_{T}} q^{2} \chi(x) \omega^{\prime}(q) d t d x \\
=\int_{\Pi_{T}} \alpha \chi(x)\left(q \omega(q)-\frac{1}{2} q^{2} \omega^{\prime}(q)\right) d t d x \\
\geq \frac{\alpha(1-\rho)}{2} \int_{\Pi_{T}} \chi(x) q^{2}(1+|q|)^{\rho} d t d x
\end{gathered}
$$

Applying the Hölder inequality, (15) and (20) give rise to

$$
\begin{aligned}
& \left|\int_{R} \chi(x) \omega(q) d x\right| \leq \int_{R} \chi(x)\left(|q|^{1+\rho}+|q|\right) d x \\
& \leq\|\chi\|_{L^{2 /(1-\rho)}(R)}\|q\|_{L^{2}(R)}^{1+\rho}+\|\chi\|_{L^{2}(R)}\|q\|_{L^{2}(R)} \\
& \leq(b-a+2)^{(1-\rho) / 2}\left\|v_{0}\right\|_{H^{1}(R)}^{1+\rho}+(b-a+2)^{1 / 2}\left\|v_{0}\right\|_{H^{1}(R)}, \\
& \left|\int_{\Pi_{T}} \alpha v \chi^{\prime}(x) \omega(q) d t d x\right| \\
& \leq \int_{\Pi_{T}} \alpha|v|\left|\chi^{\prime}(x)\right|\left(|q|^{1+\rho}+|q|\right) d t d x \\
& \leq \alpha\left\|v_{0}\right\|_{H^{1}(R)} \\
& \quad \times \int_{0}^{T}\left(\left\|\chi^{\prime}\right\|_{L^{2 /(1-\rho)}(R)}\|q\|_{L^{2}(R)}^{1+\rho}+\left\|\chi^{\prime}\right\|_{L^{2}(R)}\|q\|_{L^{2}(R)}\right) d t \\
& \leq c T\left(\left\|\chi^{\prime}\right\|_{L^{2 /(1-\rho)}(R)}\left\|v_{0}\right\|_{H^{1}(R)}^{1+\rho}+\left\|\chi^{\prime}\right\|_{L^{2}(R)}\left\|v_{0}\right\|_{H^{1}(R)}\right) .
\end{aligned}
$$

Integrating by parts, we have

$$
\int_{\Pi_{T}} \frac{\partial q}{\partial x} \chi^{\prime}(x) \omega^{\prime}(q) d t d x=-\int_{\Pi_{T}} \omega(q) \chi^{\prime \prime}(x) d t d x .
$$

From (20), (26), and the Hölder inequality, we have

$$
\begin{aligned}
& \left|\int_{\Pi_{T}} \frac{\partial q}{\partial x} \chi^{\prime}(x) \omega^{\prime}(q) d t d x\right| \leq \varepsilon \int_{\Pi_{T}}\left|\omega(q) \| \chi^{\prime \prime}(x)\right| d t d x \\
& \leq \varepsilon \int_{\Pi_{T}}\left|\chi^{\prime \prime}(x)\right|\left(|q|^{1+\rho}+|q|\right) d t d x \\
& \leq \varepsilon \int_{0}^{T}\left(\left\|\chi^{\prime \prime}\right\|_{L^{2 /(1-\rho)}(R)}\|q\|_{L^{2}(R)}^{1+\rho}+\left\|\chi^{\prime \prime}\right\|_{L^{2}(R)}\|q\|_{L^{2}(R)}\right) d t \\
& \leq \varepsilon T\left(\left\|\chi^{\prime \prime}\right\|_{L^{2 /(1-\rho)(R)}}\left\|v_{0}\right\|_{H^{1}(R)}^{1+\rho}+\left\|\chi^{\prime \prime}\right\|_{L^{2}(R)}\left\|v_{0}\right\|_{H^{1}(R)}\right) .
\end{aligned}
$$

Using (20) and Lemma 3 derives

$$
\begin{aligned}
& \varepsilon\left|\int_{\Pi_{T}}\left(\frac{\partial q}{\partial x}\right)^{2} \chi(x) \omega^{\prime \prime}(q) d t d x\right| \\
& \quad \leq 2 \gamma \varepsilon \int_{\Pi_{T}}\left(\frac{\partial q}{\partial x}\right)^{2} d t d x \leq \gamma T\left\|v_{0}\right\|_{H^{1}(R)}^{2}
\end{aligned}
$$

It follows from (20) that

$$
\begin{gathered}
\left|\int_{\Pi_{T}}\left[\beta v^{2 m}+\lambda\right] q \chi(x) \theta^{\prime}(q) d t d x\right| \\
\leq c \int_{\Pi_{T}}|\chi(x)|\left((1+\rho) q^{2}+|q|\right) d t d x \\
\leq \beta c\left((1+\rho)\|\chi\|_{L^{\infty}}\left\|v_{0}\right\|_{H^{1}(R)}^{2}\right. \\
\left.+\|\chi(x)\|_{L^{2}(R)}\left\|v_{0}\right\|_{H^{1}(R)}\right) .
\end{gathered}
$$

In fact, we have

$$
\begin{aligned}
& \left|\Lambda^{-2}\left[v^{2 m-1} v_{x}^{2}\right]_{x}\right| \\
& \quad=\left|\frac{1}{2} e^{-x} \int_{-\infty}^{x} e^{y}\left[v^{2 m-1} v_{y}^{2}\right]_{y} d y+\frac{1}{2} e^{x} \int_{x}^{\infty} e^{-y}\left[v^{2 m-1} v_{y}^{2}\right]_{y} d y\right| \\
& =\left|-\frac{1}{2} e^{-x} \int_{-\infty}^{x} e^{y} v^{2 m-1} v_{y}^{2} d y+\frac{1}{2} e^{x} \int_{x}^{\infty} v^{2 m-1} v_{y}^{2} d y\right| \\
& \quad \leq 2 \int_{-\infty}^{\infty} e^{-|x-y|}\left|v^{2 m-1}\right| v_{y}^{2} d y \\
& \leq 2\|v\|_{L^{\infty}(R)}^{2 m-1}\|v\|_{H^{1}(R)} \\
& \leq 2\left\|v_{0}\right\|_{L^{\infty}(R)}^{2 m-1}\left\|v_{0}\right\|_{H^{1}(R)} .
\end{aligned}
$$

Applying (15), the Hölder inequality, Lemma 3, and $\int_{R} e^{-|x-y|} d y=2$, we have

$$
\begin{aligned}
& \left|f(v)-\frac{\alpha}{2} v^{2}-\Lambda^{-2}\left[f(v)-\frac{\alpha}{2}\left(v^{2}-v_{x}^{2}\right)-\beta v^{2 m} v_{x}\right]\right| \\
& \leq 3\left(\|f(v)\|_{L^{\infty}(R)}+\frac{\alpha}{2}\|v\|_{L^{\infty}(R)}^{2}\right)+\frac{\alpha}{2}\|v\|_{H^{1}(R)}^{2} \\
& \quad+\beta\|v\|_{L^{\infty}(R)}^{2 m-1}\|v\|_{L^{2}(R)}\left\|v_{x}\right\|_{L^{2}(R)} \\
& \leq c,
\end{aligned}
$$

where $c$ is a constant independent of $\varepsilon$.

From (30) and (31), we know that there exists a positive constant $c$ depending on $\left\|v_{0}\right\|_{H^{1}(R)}$, but independent of $\varepsilon$, such that

$$
\left\|P_{\varepsilon}(t, x)\right\|_{L^{\infty}(R)} \leq c
$$


which results in

$$
\begin{aligned}
& \left|\int_{\Pi_{T}} P_{\varepsilon}(t, x) \chi(x) \omega^{\prime}(q) d t d x\right| \\
& \leq c \int_{\Pi_{T}}|\chi(x)|((1+\rho)|q|+1) d t d x \\
& \leq c T\left((1+\rho)\|\chi(x)\|_{L^{2}(R)}\left\|v_{0}\right\|_{H^{1}(R)}+\int_{R}|\chi(x)| d x\right) .
\end{aligned}
$$

From inequalities (22)-(29) and (33), we obtain (17).

Lemma 5. There exists a positive constant $C$ depending only on $\left\|v_{0}\right\|_{H^{1}(R)}$ and the coefficients of (1) such that

$$
\begin{gathered}
\left\|P_{\varepsilon}(t, \cdot)\right\|_{L^{\infty}(R)} \leq c, \\
\left\|P_{\varepsilon}(t, \cdot)\right\|_{L^{1}(R)} \leq c, \\
\left\|P_{\varepsilon}(t, \cdot)\right\|_{L^{2}(R)} \leq c .
\end{gathered}
$$

Proof. Writing $v(t, x)=v_{\varepsilon}(t, x)$, we get

$$
\begin{aligned}
P_{\varepsilon}(t, x)=f(v)-\frac{\alpha}{2} v^{2}-\Lambda^{-2}[ & f(v)-\frac{\alpha}{2}\left(v^{2}-v_{x}^{2}\right)-\beta v^{2 m} v_{x} \\
& \left.+2 m \beta \partial_{x}\left[v^{2 m-1} v_{x}^{2}\right]\right] .
\end{aligned}
$$

Inequality (34) is proved in Lemma 4 (see (32)). Now we prove (35). Since

$$
\begin{aligned}
& \int_{R}\left|\Lambda^{-2}\left[v^{2 m-1} v_{x}^{2}\right]_{x}\right| d x \\
& =\int_{R} \mid \frac{1}{2} e^{-x} \int_{-\infty}^{x} e^{y}\left[v^{2 m-1} v_{y}^{2}\right]_{y} d y \\
& \quad+\frac{1}{2} e^{x} \int_{x}^{\infty} e^{-y}\left[v^{2 m-1} v_{y}^{2}\right]_{y} d y \mid d x \\
& =\int_{R} \mid-\frac{1}{2} \int_{-\infty}^{x} e^{y} v^{2 m-1} v_{y}^{2} d y \\
& \quad+\frac{1}{2} \int_{x}^{\infty} v^{2 m-1} v_{y}^{2} d y \mid d x, \\
& \leq \int_{R} e^{-|x-y|}\left|v^{2 m-1}\right| v_{y}^{2} d y d x \\
& \leq 2\|v\|_{L^{\infty}(R)}^{2 m-1}\|v\|_{H^{1}(R)} \leq 2\left\|v_{0}\right\|_{L^{\infty}(R)}^{2 m-1}\left\|v_{0}\right\|_{H^{1}(R)}, \\
& \qquad \int_{R}\left|\int_{R} e^{-|x-y|}\left[f(v)-\frac{\alpha}{2} v^{2}+\frac{\alpha}{2} v_{y}^{2}-\beta v^{2 m} v_{y}\right] d y\right| d x \\
& \leq(v)-\frac{\alpha}{2} v^{2} \| \\
& \leq c_{0}\left(\left\|v_{0}^{1}\right\|_{H^{1}(R)}^{n}+\left\|v_{0}\right\|_{H^{1}(R)}^{2}\right), \\
& \leq c_{0}\|v\|_{L^{\infty}(R)}^{n-2}\|v\|_{L^{2}(R)}^{2}+\frac{\alpha}{2}\|v\|_{L^{2}(R)}^{2} \\
& \left.\leq f(v)-\frac{\alpha}{2} v^{2}+\frac{\alpha}{2} v_{x}^{2}-\beta v^{2 m} v_{x}\right] \mid d x
\end{aligned}
$$

$$
\begin{aligned}
& \leq \int_{R} e^{-|x-y|} d x \int_{R}\left|f(v)-\frac{\alpha}{2} v^{2}+\frac{\alpha}{2} v_{y}^{2}-\beta v^{2 m} v_{y}\right| d y \\
& \leq 2 c_{0}\left(\left\|v_{0}\right\|_{H^{1}(R)}^{2}+\left\|v_{0}\right\|_{H^{1}(R)}^{n}+\|v\|_{H^{1}(R)}^{2}+\|v\|_{L^{\infty}(R)}^{2 m-1}\|v\|_{H^{1}(R)}^{2}\right) \\
& \leq c_{0}\left(\left\|v_{0}\right\|_{H^{1}(R)}^{2}+\left\|v_{0}\right\|_{H^{1}(R)}^{n}+\left\|v_{0}\right\|_{H^{1}(R)}^{2 m+1}\right),
\end{aligned}
$$

we know that (35) holds.

Applying the Tonelli theorem, (34), and (35), we get

$$
\left\|P_{\varepsilon}(t, \cdot)\right\|_{L^{2}(R)}^{2} \leq\left\|P_{\varepsilon}(t, \cdot)\right\|_{L^{\infty}}\left\|P_{\varepsilon}(t, \cdot)\right\|_{L^{1}(R)} \leq c .
$$

The proof of Lemma 5 is completed.

Lemma 6. Assume that $v_{\varepsilon}=v_{\varepsilon}(t, x)$ is the unique solution of (10). For an arbitrary $T>0$, there exists a positive constant $C$ depending only on $\left\|v_{0}\right\|_{H^{1}(R)}$ and the coefficients of (1) such that the following one-sided $L^{\infty}$ norm estimate on the first order spatial derivative holds:

$$
\frac{\partial v_{\varepsilon}(t, x)}{\partial x} \leq \frac{4}{\alpha t}+C, \quad \text { for }(t, x) \in[0, \infty) \times R .
$$

Proof. From (16) and Lemma 5, we know that there exists a positive constant $C$ depending only on $\left\|v_{0}\right\|_{H^{1}(R)}$ and the coefficients of $(1)$ such that $\left\|P_{\varepsilon}(t, x)\right\|_{L^{\infty}(R)} \leq C$. Therefore,

$$
\begin{aligned}
\frac{\partial q_{\varepsilon}}{\partial t} & +\alpha v_{\varepsilon} \frac{\partial q_{\varepsilon}}{\partial x}+\frac{\alpha}{2} q_{\varepsilon}^{2}+\beta\left(v_{\varepsilon}\right)^{2 m} q_{\varepsilon}+\lambda q_{\varepsilon}-\varepsilon \frac{\partial^{2} q_{\varepsilon}}{\partial x^{2}} \\
& =P_{\varepsilon}(t, x) \leq C .
\end{aligned}
$$

Let $g=g(t)$ be the solution of

$$
\begin{aligned}
& \frac{d g}{d t}+\frac{\alpha}{2} g^{2}+\beta\left(v^{*}\right)^{2 m} g+\lambda g=C, \quad t>0, \\
& g(0)=\left\|\frac{\partial v_{\varepsilon, 0}}{\partial x}\right\|_{L^{\infty}(R)},
\end{aligned}
$$

where $v_{\varepsilon}^{*}$ is the value of $v_{\varepsilon}(t, x)$ when $\sup _{x \in R} q_{\varepsilon}(t, x)=g(t)$. From the comparison principle for parabolic equations, we get

$$
q_{\varepsilon}(t, x) \leq g(t)
$$

Using (15) and suitably choosing $C_{1}$, we have $-\beta\left(u^{*}\right)^{2 m} g \leq(\alpha / 8) g^{2}+C_{1}$ and $-\lambda g \leq(\alpha / 8) g^{2}+C_{1}$. Furthermore, we have

$$
\begin{aligned}
\frac{d g}{d t} & =C-\frac{\alpha}{2} g^{2}-\beta\left(v^{*}\right)^{2 m} g-\lambda g \\
& \leq C-\frac{\alpha}{2} g^{2}+\frac{\alpha}{4} g^{2}+2 C_{1} \leq C-\frac{\alpha}{4} g^{2}+2 C_{1} .
\end{aligned}
$$

Setting $M_{0}=C+2 C_{1}$, we obtain

$$
\frac{d g}{d t}+\frac{\alpha}{4} g^{2} \leq M_{0} .
$$


Letting $K(t)=4 / \alpha t+\sqrt{(4 / \alpha) M_{0}}$, we have $d K(t) / d t+$ $(\alpha / 4) K^{2}(t)-M_{0}=4 \sqrt{M_{0} / \alpha} / t>0$. From the comparison principle for ordinary differential equations, we get $g(t) \leq$ $K(t)$ for all $t>0$. Therefore, by this and (43), the estimate (40) is proved.

Lemma 7. There exists a sequence $\left\{\varepsilon_{j}\right\}_{j \in N}$ tending to zero and a function $v \in L^{\infty}\left([0, \infty) ; H^{1}(R)\right) \cap H^{1}([0, T] \times R)$ such that, for each $T>0$, it holds that

$$
\begin{gathered}
v_{\varepsilon_{j}} \rightarrow v \quad \text { in } H^{1}([0, T] \times R), \quad \text { for each } T>0, \\
v_{\varepsilon_{j}} \longrightarrow v \quad \text { in } L_{\mathrm{loc}}^{\infty}([0, \infty) \times R),
\end{gathered}
$$

where $v_{\varepsilon}=v_{\varepsilon}(t, x)$ is the unique solution of (10).

The proof of this lemma is fully similar to that of Lemma 5.2 in [6]. Here we omit its proof.

Lemma 8. There exists a sequence $\left\{\varepsilon_{j}\right\}_{j \in N}$ tending to zero and a function $P \in L^{\infty}([0, \infty) \times R)$ such that for each $1<p<\infty$

$$
P_{\varepsilon_{j}} \longrightarrow P \quad \text { strongly in } L_{\text {loc }}^{p}([0, \infty) \times R) \text {. }
$$

Proof. Using the same arguments presented in Coclite et al. [6], we obtain $\int_{R}\left|d P_{\varepsilon} / d t\right| d x \leq c$ which together with Lemma 5 derives that (47) holds.

In this paper we use overbars to denote weak limits which are taken in $L_{\mathrm{loc}}^{p}([0, \infty) \times R)$ with $1<p<3$.

Lemma 9. There exists a sequence $\left\{\varepsilon_{j}\right\}_{j \in N}$ tending to zero and two functions $q \in L_{\mathrm{loc}}^{p}([0, \infty) \times R), \overline{q^{2}} \in L_{\mathrm{loc}}^{r}([0, \infty) \times R)$ such that

$$
\begin{aligned}
& q_{\varepsilon_{j}} \rightarrow q \quad \text { in } L_{\mathrm{loc}}^{p}([0, \infty) \times R), \\
& q_{\varepsilon_{j}} \stackrel{\star}{\rightarrow} q \text { in } L_{\mathrm{loc}}^{\infty}\left([0, \infty) ; L^{2}(R)\right), \\
& q_{\varepsilon_{j}}^{2} \rightarrow \overline{q^{2}} \quad \text { in } L_{\mathrm{loc}}^{r}([0, \infty) \times R),
\end{aligned}
$$

for each $1<p<3$ and $1<r<3 / 2$. Moreover,

$q^{2}(t, x) \leq \overline{q^{2}}(t, x) \quad$ for almost every $(t, x) \in[0, \infty) \times R$,

$$
\frac{\partial v}{\partial x}=q \text { in the sense of distributions on }[0, \infty) \times R \text {. }
$$

Proof. Using Lemmas 3 and 4 derives that (48) and (49) hold. It follows from (49) that (50) holds. From Lemma 7 and (48), we know that (51) is valid.

Using (48), for any convex function $\psi \in C^{1}(R)$ with $\psi^{\prime}$ being bounded and Lipschitz continuous on $R$ and for any $1<p<3$, we conclude that

$$
\begin{gathered}
\psi\left(q_{\varepsilon}\right) \rightarrow \overline{\psi(q)} \quad \text { in } L_{\mathrm{loc}}^{p}([0, \infty) \times R), \\
\psi\left(q_{\varepsilon}\right) \stackrel{\star}{\rightarrow} \overline{\psi(q)} \quad \text { in } L_{\mathrm{loc}}^{\infty}\left([0, \infty) ; L^{2}(R)\right) .
\end{gathered}
$$

Multiplying (16) by $\psi^{\prime}\left(q_{\varepsilon}\right)$ yields

$$
\begin{aligned}
\frac{\partial}{\partial t} \psi & \left(q_{\varepsilon}\right)+\alpha \frac{\partial}{\partial x}\left(v_{\varepsilon} \psi\left(q_{\varepsilon}\right)\right)-\varepsilon \frac{\partial^{2}}{\partial x^{2}} \psi\left(q_{\varepsilon}\right) \\
& +\varepsilon \psi^{\prime \prime}\left(q_{\varepsilon}\right)\left(\frac{\partial q_{\varepsilon}}{\partial x}\right)^{2}=\alpha q_{\varepsilon} \psi\left(q_{\varepsilon}\right)-\frac{\alpha}{2} \psi^{\prime}\left(q_{\varepsilon}\right) q_{\varepsilon}^{2} \\
& -\left[\beta\left(v_{\varepsilon}\right)^{2 m}+\lambda\right] q_{\varepsilon} \psi^{\prime}\left(q_{\varepsilon}\right)+P_{\varepsilon}(t, x) \psi^{\prime}\left(q_{\varepsilon}\right) .
\end{aligned}
$$

Lemma 10. For the convex $\psi \in C^{1}(R)$ with $\psi^{\prime}$ being bounded and Lipschitz continuous on $R$, it has

$$
\begin{aligned}
& \overline{\frac{\partial \psi(q)}{\partial t}}+\frac{\partial}{\partial x}(\alpha v \overline{\psi(q)}) \\
& \leq \alpha \overline{q \psi(q)}-\frac{\alpha}{2} \overline{\psi^{\prime}(q) q^{2}} \\
& -\left[\beta v^{2 m}+\lambda\right] \overline{q \psi^{\prime}(q)}+P(t, x) \overline{\psi^{\prime}(q)}
\end{aligned}
$$

in the sense of distributions on $[0, \infty) \times R$. Here $\overline{q \psi(q)}$ and $\overline{\psi^{\prime}(q) q^{2}}$ denote the weak limits of $q_{\varepsilon} \psi\left(q_{\varepsilon}\right)$ and $q_{\varepsilon}^{2} \psi^{\prime}\left(q_{\varepsilon}\right)$ in $L_{\text {loc }}^{r}([0, \infty) \times R), 1<r<3 / 2$, respectively.

Proof. In (53), by the convexity of $\psi$, (15), Lemmas 7, 8, and 9 , taking limit for $\varepsilon \rightarrow 0$ gives rise to the desired result.

Remark 11. Using Lemma 9, we know that

$$
\begin{aligned}
& q=q_{+}+q_{-}=\overline{q_{+}}+\overline{q_{-}}, \quad q^{2}=\left(q_{+}\right)^{2}+\left(q_{-}\right)^{2}, \\
& \overline{q^{2}}=\overline{\left(q_{+}\right)^{2}}+\overline{\left(q_{-}\right)^{2}}
\end{aligned}
$$

almost everywhere in $[0, \infty) \times R$, where $\xi_{+}:=$ $\xi_{\chi[0,+\infty)}(\xi), \xi_{-}:=\xi_{\chi(-\infty, 0]}(\xi)$ for $\xi \in R$. From Lemma 6 and (48), we have

$$
q_{\varepsilon}(t, x), \quad q(t, x) \leq \frac{4}{\alpha t}+C, \quad \text { for } t>0, x \in R,
$$

where $C$ is a constant depending only on $\left\|v_{0}\right\|_{H^{1}(R)}$ and the coefficients of (1).

Lemma 12. The identity

$$
\frac{\partial q}{\partial t}+\frac{\partial}{\partial x}(\alpha v q)=\frac{\alpha}{2} \overline{q^{2}}-\left[\beta v^{2 m}+\lambda\right] q+P(t, x)
$$

holds in the sense of distributions on $[0, \infty) \times R$.

Proof. Applying (16), Lemmas 7 and 8, (48), (49), and (51), the conclusion (57) holds by taking limit for $\varepsilon \rightarrow 0$ in (16).

Lemma 13. If $\psi \in C^{1}(R)$ with $\psi \in L^{\infty}(R)$, then the identity

$$
\begin{aligned}
\frac{\partial \psi(q)}{\partial t} & +\frac{\partial}{\partial x}(\alpha v \psi(q)) \\
= & \alpha q \psi(q)+\left(\frac{\alpha}{2} \overline{q^{2}}-\alpha q^{2}\right) \psi^{\prime}(q) \\
& -\left[\beta v^{2 m}+\lambda\right] q \psi^{\prime}(q)+P(t, x) \psi^{\prime}(q)
\end{aligned}
$$

holds in the sense of distributions on $[0, \infty) \times R$. 
Proof. Let $\left\{\omega_{\delta}\right\}_{\delta}$ be a family of mollifiers defined on $R$. Consider $q_{\delta}(t, x):=\left(q(t, \cdot) \star \omega_{\delta}\right)(x)$ where the $\star$ is the convolution with respect to $x$ variable. Multiplying (57) by $\psi^{\prime}\left(q_{\delta}\right)$ gives rise to

$$
\begin{aligned}
& \frac{\partial \psi\left(q_{\delta}\right)}{\partial t} \\
& =\psi^{\prime}\left(q_{\delta}\right) \frac{\partial q_{\delta}}{\partial t} \\
& =\psi^{\prime}\left(q_{\delta}\right)\left[\frac{\alpha}{2} \overline{q^{2}} \star \omega_{\delta}-\left[\beta v^{2 m}+\lambda\right] q_{\delta}\right. \\
& +P(t, x) \star \omega_{\delta}-\alpha q^{2} \\
& \left.\star \omega_{\delta}-\alpha v \frac{\partial q}{\partial x} \star \omega_{\delta}\right], \\
& \frac{\partial}{\partial x}\left(\alpha v \psi\left(q_{\delta}\right)\right)=\alpha q \psi\left(q_{\delta}\right)+\alpha v \psi^{\prime}\left(q_{\delta}\right)\left(\frac{\partial q_{\delta}}{\partial x}\right) .
\end{aligned}
$$

Applying the boundedness of $\psi, \psi^{\prime}$ and letting $\delta \rightarrow 0$ in (59), we derive that (58) holds.

\section{Strong Convergence of $q_{\varepsilon}$ and Existence of Global Weak Solutions}

Using the methods in [5] or [6], in this section, we will prove that the weak convergence of $q_{\varepsilon}$ in (48) is strong convergence. This results in the existence of global weak solutions for problem (10).

Lemma 14. Assume that $v_{0} \in H^{1}(R)$. Then

$$
\lim _{t \rightarrow 0} \int_{R} q^{2}(t, x) d x=\lim _{t \rightarrow 0} \int_{R} \overline{q^{2}}(t, x) d x=\int_{R}\left(\frac{\partial v_{0}}{\partial x}\right)^{2} d x
$$

Lemma 15. If $v_{0} \in H^{1}(R)$, for constant $M>0$, then

$$
\lim _{t \rightarrow 0} \int_{R}\left(\overline{\psi_{M}^{ \pm}(q)}(t, x)-\psi_{M}^{ \pm}(q(t, x))\right) d x=0
$$

where

$$
\psi_{M}(\xi):= \begin{cases}\frac{1}{2} \xi^{2}, & \text { if }|\xi| \leq M, \\ M|\xi|-\frac{1}{2} M^{2}, & \text { if }|\xi|>M,\end{cases}
$$

and $\psi_{M}^{+}(\xi):=\psi_{M}(\xi) \chi_{[0,+\infty)}(\xi), \psi_{M}^{-}(\xi):=\psi_{M}(\xi) \chi_{(-\infty, 0]}(\xi)$ $\xi \in R$.
Lemma 16. Let constant $M>0$. Then for each $\xi \in R$

$$
\begin{aligned}
& \psi_{M}(\xi)=\frac{1}{2} \xi^{2}-\frac{1}{2}(M-|\xi|)^{2} \chi_{(-\infty,-M) \cap(M, \infty)}(\xi) \\
& \psi_{M}^{\prime}(\xi) \xi=\xi+(M-|\xi|) \operatorname{sign}(\xi) \chi_{(-\infty,-M) \cap(M, \infty)}(\xi) \\
& \psi_{M}^{+}(\xi)=\frac{1}{2}\left(\xi_{+}\right)^{2}-\frac{1}{2}(M-\xi)^{2} \chi_{(M, \infty)}(\xi) \\
& \left(\psi_{M}^{+}\right)^{\prime}(\xi)=\xi_{+}+(M-\xi) \chi_{(M, \infty)}(\xi) \\
& \psi_{M}^{-}(\xi)=\frac{1}{2}\left(\xi_{-}\right)^{2}-\frac{1}{2}(M+\xi)^{2} \chi_{(-\infty,-M)}(\xi) \\
& \left(\psi_{M}^{-}\right)^{\prime}(\xi)=\xi_{-}-(M+\xi) \chi_{(-\infty,-M)}(\xi)
\end{aligned}
$$
or [6].

The proofs of Lemmas 14, 15, and 16 can be found in [5]

Lemma 17. Assume that $v_{0} \in H^{1}(R)$. Then for almost all $t>0$

$$
\begin{aligned}
& \frac{1}{2} \int_{R}\left(\overline{\left(q_{+}\right)^{2}}-q_{+}^{2}\right)(t, x) d x \\
& \quad \leq \int_{0}^{t} \int_{R} P(s, x)\left[\overline{q_{+}}(s, x)-q_{+}(s, x)\right] d s d x .
\end{aligned}
$$

Lemma 18. For any $t>0$ and constant $M>0$, it holds that

$$
\begin{aligned}
\int_{R}\left(\overline{\psi_{M}^{-}(q)}-\psi_{M}^{-}(q)\right)(t, x) d x \\
\leq \frac{\alpha M^{2}}{2} \int_{0}^{t} \int_{R} v \overline{(M+q) \chi_{(-\infty,-M)}(q)} d s d x \\
\quad-\frac{\alpha M^{2}}{2} \int_{0}^{t} \int_{R} v(M+q) \chi_{(-\infty,-M)}(q) d s d x \\
+\alpha M \int_{0}^{t} \int_{R} v\left[\overline{\psi_{M}^{-}(q)}-\psi_{M}^{-}(q)\right] d s d x \\
\quad+\frac{\alpha M}{2} \int_{0}^{t} \int_{R} v\left(\overline{q_{+}^{2}}-q_{+}^{2}\right) d s d x \\
+\int_{0}^{t} \int_{R} P(t, x)\left(\overline{\left(\psi_{M}^{-}\right)^{\prime}(q)}-\left(\psi_{M}^{-}\right)^{\prime}(q)\right) d s d x .
\end{aligned}
$$

Using the same techniques in [5] or [6], because of (54), (58), and Lemma 6, we can prove Lemmas 17 and 18. Here we omit their proofs.

Lemma 19. It holds that

$\overline{q^{2}}=q^{2} \quad$ almost everywhere in $[0, \infty) \times(-\infty, \infty)$.

Directly applying the approaches in [5] or [6] and Lemmas 17 and 18, we obtain that Lemma 19 holds. Here we omit its proof.

Proof of the Main Result. From (9), (11), and Lemma 7, we conclude that conditions (a) and (b) in Definition 1 are satisfied. We need to prove (c). Using Lemma 19, we get

$$
q_{\varepsilon} \longrightarrow q \quad \text { in } L_{\text {loc }}^{2}([0, \infty) \times R) \text {. }
$$


From Lemma 7, (47), and (67), we know that $v$ is a distributional solution to problem (3). The inequalities (6) and (7) are consequences of Lemmas 4 and 6 . The proof is completed.

\section{Conflict of Interests}

The authors declare that there is no conflict of interests regarding the publication of this paper.

\section{Authors' Contribution}

The paper is a joint work of three authors who contributed equally to the final version of the paper. All the authors read and approved the final paper.

\section{References}

[1] R. Camassa and D. D. Holm, "An integrable shallow water equation with peaked solitons," Physical Review Letters, vol. 71, no. 11, pp. 1661-1664, 1993.

[2] H. H. Dai, "Exact travelling-wave solutions of an integrable equation arising in hyperelastic rods," Wave Motion, vol. 28, no. 4, pp. 367-381, 1998.

[3] A. Constantin and J. Escher, "Global weak solutions for a shallow water equation," Indiana University Mathematics Journal, vol. 47, no. 4, pp. 1527-1545, 1998.

[4] A. Constantin and L. Molinet, "Global weak solutions for a shallow water equation," Communications in Mathematical Physics, vol. 211, no. 1, pp. 45-61, 2000.

[5] Z. Xin and P. Zhang, "On the weak solutions to a shallow water equation," Communications on Pure and Applied Mathematics, vol. 53, no. 11, pp. 1411-1433, 2000.

[6] G. M. Coclite, H. Holden, and K. H. Karlsen, "Global weak solutions to a generalized hyperelastic-rod wave equation," SIAM Journal on Mathematical Analysis, vol. 37, no. 4, pp. 10441069, 2005.

[7] S. Lai and Y. Wu, "The local well-posedness and existence of weak solutions for a generalized Camassa-Holm equation," Journal of Differential Equations, vol. 248, no. 8, pp. 2038-2063, 2010.

[8] Z. Sheng, S. Lai, Y. Ma, and X. Luo, "The $H^{1}(R)$ space global weak solutions to the weakly dissipative Camassa-Holm equation," Abstract and Applied Analysis, vol. 2012, Article ID 693010, 21 pages, 2012.

[9] A. Bressan and A. Constantin, "Global conservative solutions of the Camassa-Holm equation," Archive for Rational Mechanics and Analysis, vol. 183, no. 2, pp. 215-239, 2007.

[10] G. M. Coclite, H. Holden, and K. H. Karlsen, "Wellposedness for a parabolic-elliptic system," Discrete and Continuous Dynamical Systems A, vol. 13, no. 3, pp. 659-682, 2005.

[11] A. Constantin and J. Escher, "Wave breaking for nonlinear nonlocal shallow water equations," Acta Mathematica, vol. 181, no. 2, pp. 229-243, 1998.

[12] Y. Zhou, "Blow-up of solutions to a nonlinear dispersive rod equation," Calculus of Variations and Partial Differential Equations, vol. 25, no. 1, pp. 63-77, 2006. 


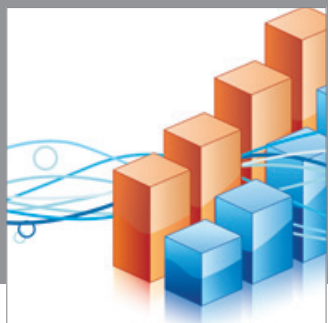

Advances in

Operations Research

mansans

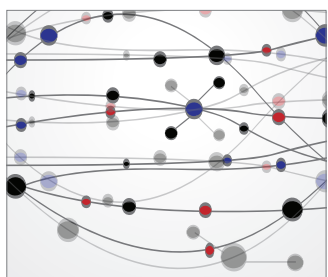

The Scientific World Journal
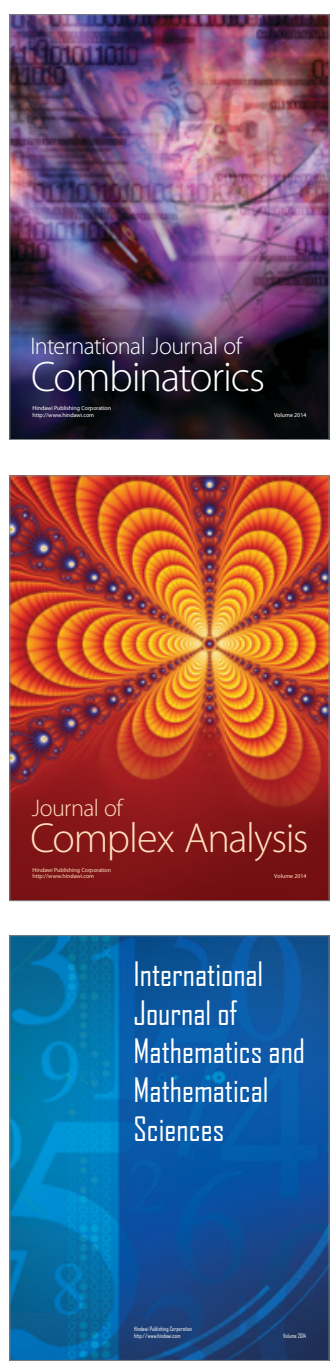
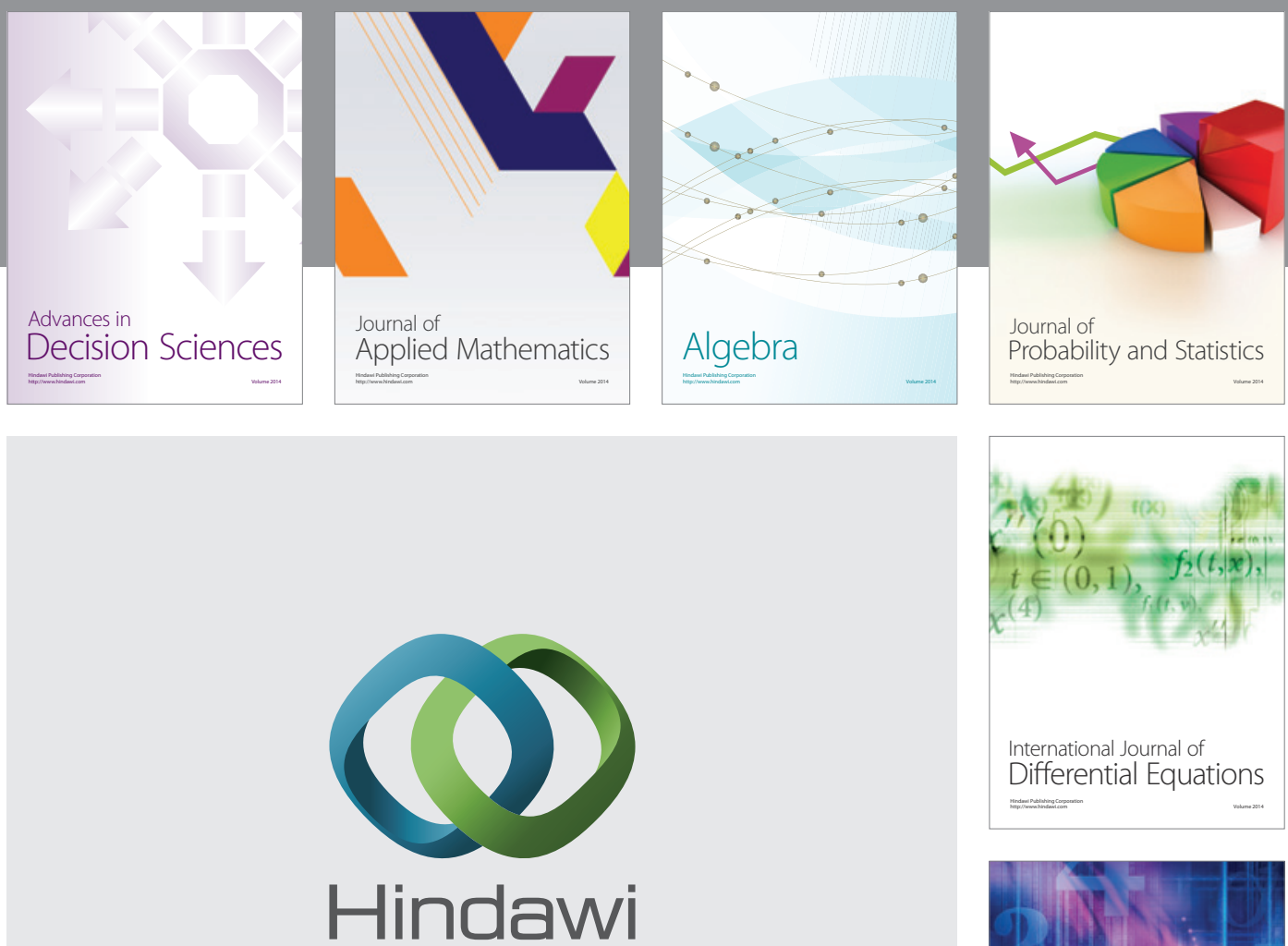

Submit your manuscripts at http://www.hindawi.com
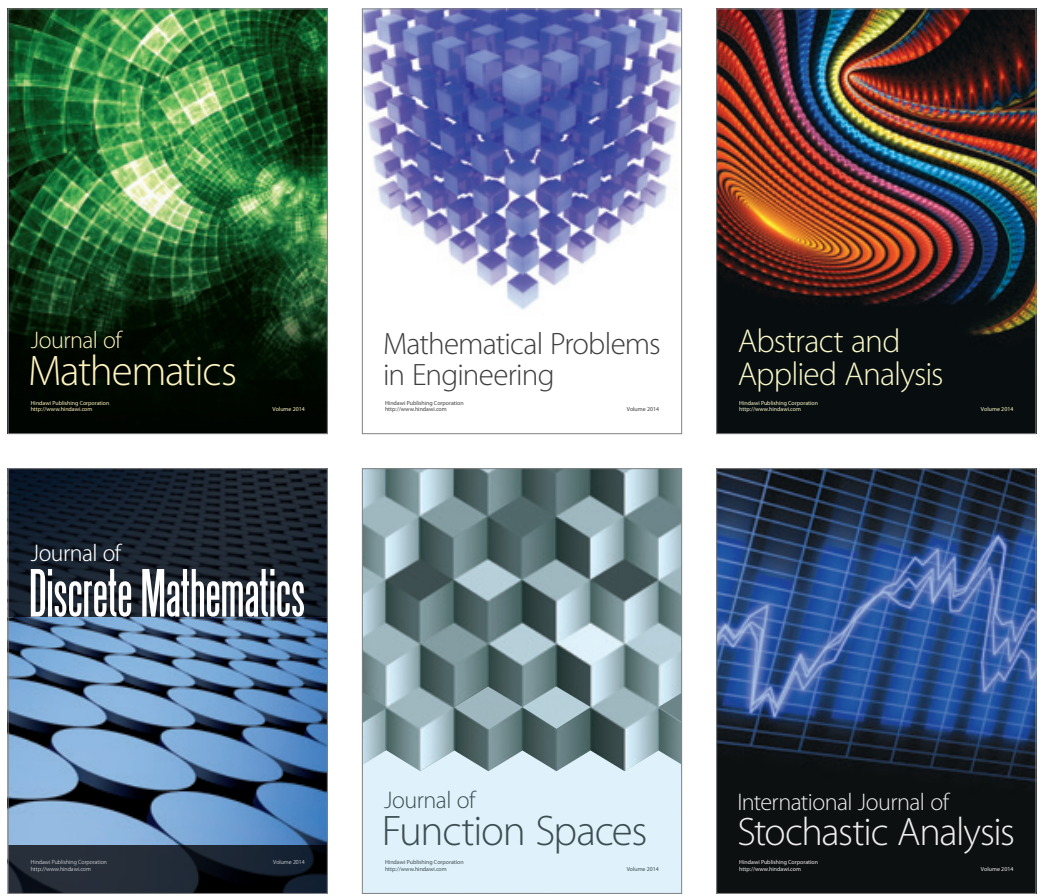

Journal of

Function Spaces

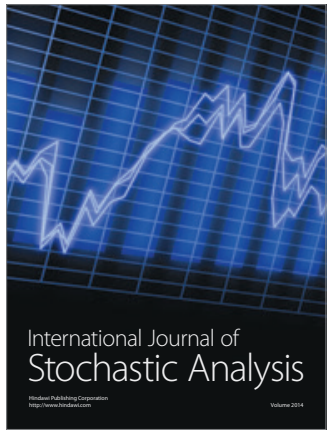

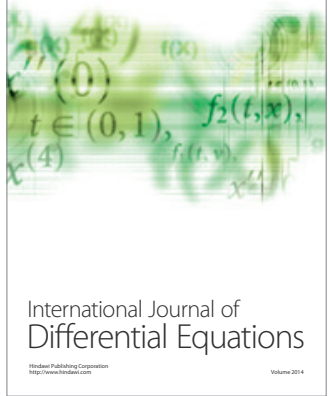
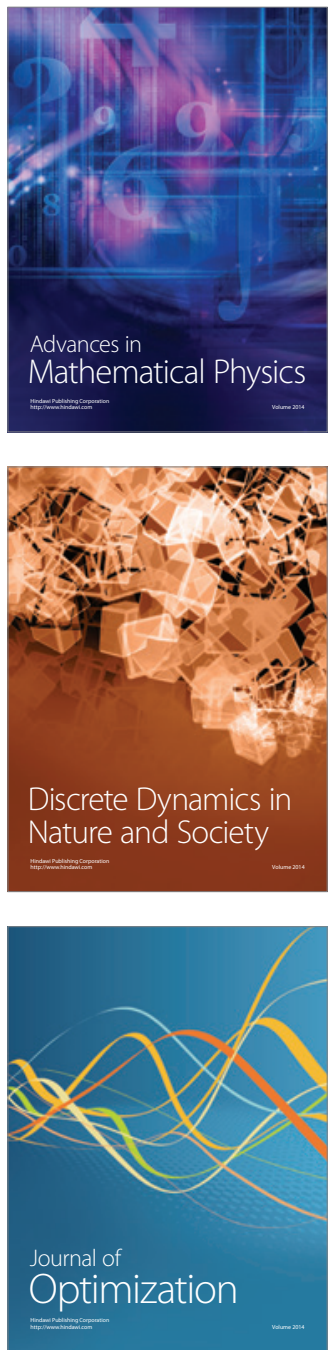